\title{
Urachal Diverticulum
}

National Cancer Institute

\section{Source}

National Cancer Institute. Urachal Diverticulum. NCI Thesaurus. Code C123254.

A condition in which the urachus fails to close proximal to the bladder, resulting in a nondetrusor, blind-ending pouch at the dome of the bladder. 